\title{
ASSESSING TEXT READING AND TEXT ENTRY WHILE DRIVING USING THE VISUAL OCCLUSION TECHNIQUE
}

\author{
Mahtab Ghazizadeh ${ }^{1}$, John D. Lee ${ }^{1}$, Yiyun Peng ${ }^{2} \&$ Linda Ng Boyle ${ }^{2}$ \\ ${ }^{1}$ University of Wisconsin-Madison, Madison, Wisconsin, USA \\ ${ }^{2}$ University of Washington, Seattle, Washington, USA \\ Email: ghazizadeh@wisc.edu
}

\begin{abstract}
Summary: This study estimated the time drivers spend completing text reading and text entry tasks of varying difficulty levels using visual occlusion to mimic the timesharing between driving and interacting with text. The findings showed that text entry took longer than text reading and task time increased with longer text length. In the occlusion condition, the total task time with vision unoccluded was shorter than the task time in the static condition, although this finding was not consistent across reading and entry. Ambient text (irrelevant text surrounding the text of interest) had no effect on time on task. These results should be considered in light of the acceptable limits for time on task and can inform the design of invehicle systems that require text reading or entry.
\end{abstract}

\section{INTRODUCTION}

Distracted driving has always been a potential threat to traffic safety. However, the everincreasing presence of cellphones, navigation systems, and infotainment systems in cars has underscored the increasing demands of non-driving related tasks on the drivers. This trend necessitates efforts to assess the effect of distractions on driving performance, with the goal of informing the design of safer in-vehicle technologies. One aspect of such efforts concerns the way drivers share their attention between the secondary task and the vehicle control task.

Visual occlusion is a surrogate driving task that mimics the back-and-forth eye glances to and from the roadway by providing alternating vision and occlusion intervals (Foley, 2008). The underlying theory and basic elements of this technique were first introduced by Senders et al. (1967) to demonstrate that drivers can be modeled as intermittently sampling and responding to roadway information, rather than as servo mechanisms that continuously respond to the road. As such, the occlusion technique is an apt surrogate for driving when assessing the attentional demand of in-vehicle information systems and is a widely accepted technique (see ISO 16673 (2007) for experimental design guidelines for using the occlusion technique).

Text reading and text entry are two tasks commonly performed while driving, particularly given the recent advances in smartphones and navigation systems (Lansdown, 2012). Using the occlusion technique to examine these two tasks can reveal their potential to affect drivers' attention to the road-longer task time indicates greater competing demands for drivers' attention. In this study, it was hypothesized that text entry would take longer than text reading. It was further hypothesized that longer texts and the presence of ambient text would further increase task time. Ambient text (background text that is not task relevant) may add clutter to the display and may create even greater demand on the text reading or entry task. The findings are discussed in the context of designing in-vehicle systems that involve text reading or text entry and can guide efforts to measure the visual demand posed by such systems. 


\section{METHODS}

The total duration of time spent on the task while vision was not occluded (i.e., Total Shutter Open Time [TSOT]) was used as the estimate of the time that would be dedicated to the text interaction task while driving. The design of the experiment followed the guidelines in ISO 16673.

\section{Participants}

Twenty-eight participants (14 men and 14 women) from the Madison, WI area in four age groups (18-24, 25-39, 40-54, and 55-75 years old; seven in each age group) participated in this study. Participants possessed a valid driver's licenses and drove a minimum of 7,000 miles per year. They were native English speakers, in good general health, and were comfortable using computers, touchscreens, and communicating via text messages. The study took 1-1.5 hours for each participant and participants were compensated \$20 per hour.

\section{Independent Variables}

The study was a mixed factorial, complete block design with 3 within-subject independent variables: task type (2 levels), task length (3 levels), and ambient text (2 levels). As such, there were 12 different test conditions for each participant. Each condition was repeated three times during the occlusion trials and three times during the static (no occlusion) trials for a total of 84 replications (28 participants x 3 replications). Two task types were considered: text entry, which involved manually entering text using key presses, and text reading, which involved reading static (non-scrolling) text. Three text lengths (short, medium, and long) were examined for each task type. For text entry, the three levels were 4, 6, and 12 characters. The words were based on street names that are found in the U.S. road database files. The text reading condition included character strings that are typically observed on changeable message signs and the three levels of task length were 20-40 (short), 60-80 (medium), and 120-140 (long) characters. Ambient text was considered in two levels: ambient text present and absent. Target text (for reading and entry) was displayed in a random row of the screen, and was identified with a box (Figure 1).
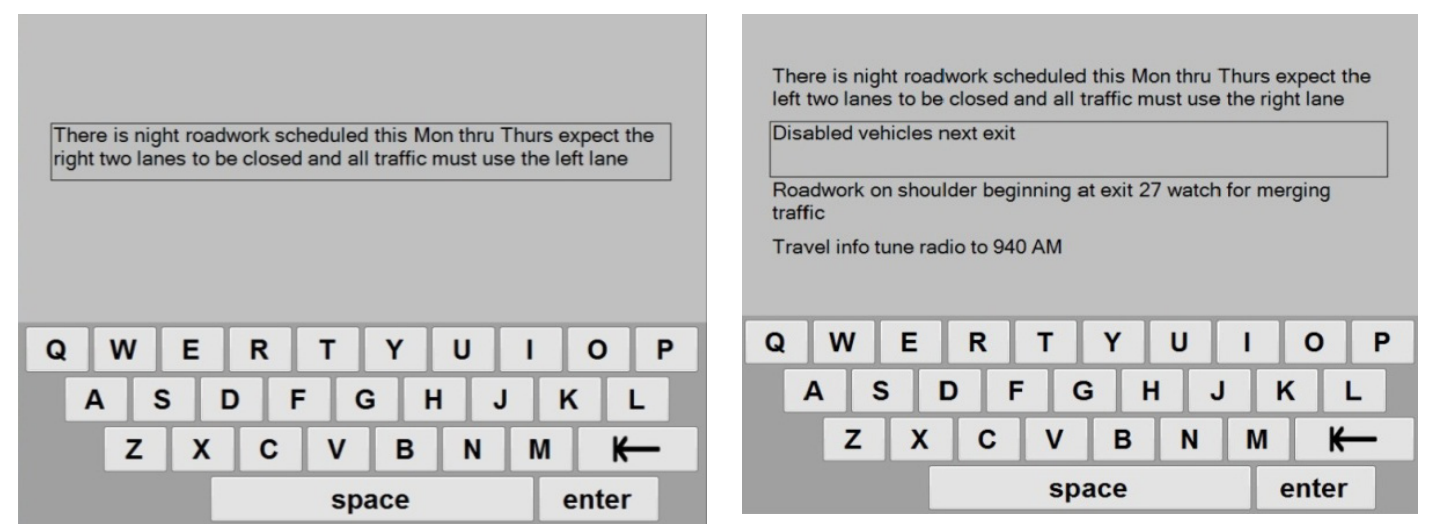

Figure 1. Examples of screens without ambient text (left) and with ambient text (right) for reading tasks

To minimize carry-over and training effects between conditions, and as recommended in the experimental design outlined in ISO 16673 (2007), two trial orders were considered: (1) 
occlusion then static and (2) static then occlusion. Fifty percent of participants received trial order one and the other 50\% received order two (randomly assigned, balanced between age groups). Data from the occlusion trials are the focus of this paper.

\section{Apparatus}

CogLens occlusion goggles (Figure 2 (a)) were used in the experiment and the participants sat in the driver's seat of a Ford Fusion vehicle simulator cab while working on tasks (Figure 2 (b)). The occlusion goggles were integrated into the text reading and text entry tasks so that the shutter close and shutter open intervals were controlled according to the ISO 16673 guidelines, i.e., $1.5 \mathrm{sec}$ vision: $1.5 \mathrm{sec}$ occlusion cycles (ISO, 2007) and the resulting data were logged with the task performance data. A 7" touchscreen display with QWERTY keyboard was used for text input and text reading tasks. The keyboard only contained keys needed for the tasks, i.e., capital alpha characters, backspace, enter, and space bar (no symbols, shift, or number pads). The keyboard had no type-ahead feature.

(a)

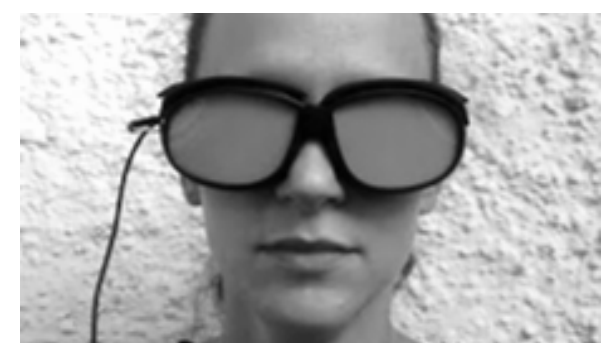

Figure 2. (a) CogLens occlusion goggles, (b) The experimental setting (b)

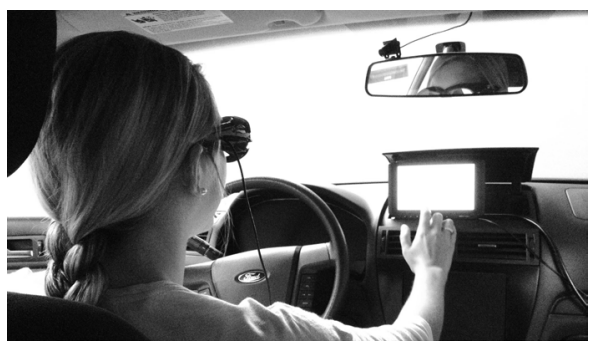

\section{Procedures}

After an IRB-approved consent form was signed, participants were trained on how to perform the tasks and how the occlusion goggles worked. Participants then proceeded to the main part of the study and completed four trials: two static and two occlusion trials (in random order). Each trial took about 8 to 15 minutes. During each trial (static or occlusion), participants were given 18 tasks: 9 text reading and 9 text entry, in a random order. Text entry tasks started with an auditory message that indicated a word to the participant to enter. The participant would then enter the word using the keyboard on the touchscreen and press ENTER when done. For text reading tasks, an audio tone prompted the participant to read the target phrase (displayed in a box). The participant read the phrase and pressed ENTER when done. An auditory statement was then provided and participants had to select whether the statement they just heard was TRUE or FALSE by pressing one of the keys (T, F) on the touchscreen.

\section{Data Analysis}

The dependent variable was Total Shutter Open Time (TSOT), the metric for time spent on task. TSOT is defined as the total time during which vision is not occluded while working on a task, and as such is considered as the surrogate measure of the duration of visual attention needed for completing a task in interrupted steps (Foley, 2008). For each task, TSOT was computed from the moment the task began to the time the participant pressed ENTER. Independent variables included within-subject factors task type, task length, and ambient text, and between-subject 
factors gender and age group. A repeated measures ANOVA (in R2.12.1, package 'nlme') was conducted to examine the relationship between the independent variables and TSOT. TSOT was $\log$ transformed in order to meet the ANOVA assumptions. A variety of models that considered different combinations of predictors and interactions were tested and the model with the lowest AIC value was selected.

\section{RESULTS}

The mean age in the four age groups were $21.3(S D=0.8), 30.4(S D=4.0), 46.6(S D=3.6)$, and $61.9(S D=35.6)$ years, respectively. There were 3 males and 4 females in each of the age groups 18-24 and 40-54 and 4 males and 3 females in each of the age groups 25-39 and 55-75. All participants had at least a high school diploma, with $9(32.1 \%)$ having some college education, $12(42.9 \%)$ having a 4-year college degree, and 4 (14.3\%) having a Master's degree or higher. The mean age of obtaining the first driver's license was 16.2 years $(S D=0.6$ years).

Table 1 shows the total task time for static trials and TSOT for occlusion trials for each task level. Mean TSOT increases as the task length increases, for both text entry and reading, and TSOTs are higher for the entry tasks compared to reading tasks of the same length level. The standard deviations follow a similar trend. For each task type and length, TSOTs are very similar with and without ambient text. The results for static trials follow a similar trend; however, total task times for static trials are generally longer than TSOTs for occlusion trials. The average static to occlusion task time ratio is 1.28 for text entry and 1.33 for text reading. This difference in ratios shows that the intermittent sampling enforced by the occlusion technique reveals the demands of text interaction more effectively than the static trials.

Table 1. Description of Total Task Time in static trials and TSOT in occlusion trials

\begin{tabular}{|c|c|c|c|c|c|c|c|c|}
\hline \multirow[b]{2}{*}{ Task Level } & \multirow[b]{2}{*}{ Length } & \multirow[b]{2}{*}{ Ambient } & \multicolumn{3}{|c|}{ Static Condition: Total Task Time (sec) } & \multicolumn{3}{|c|}{ Occlusion Condition: TSOT (sec) } \\
\hline & & & Mean & $\begin{array}{r} \pm 95 \% \text { CI of } \\
\text { mean }\end{array}$ & $\begin{array}{r}\text { Inter- } \\
\text { subject } S D\end{array}$ & Mean & $\begin{array}{r} \pm 95 \% \text { CI of } \\
\text { mean }\end{array}$ & $\begin{array}{r}\text { Inter- } \\
\text { subject } S D\end{array}$ \\
\hline \multirow{6}{*}{ Entry } & \multirow{2}{*}{ Short } & Yes & 9.66 & {$[8.79,10.52]$} & 2.27 & 6.95 & {$[6.43,7.47]$} & 2.62 \\
\hline & & No & 9.40 & {$[8.69,10.10]$} & 2.99 & 6.96 & {$[6.46,7.45]$} & 1.87 \\
\hline & \multirow{2}{*}{ Med } & Yes & 11.07 & {$[10.26,11.88]$} & 2.99 & 8.90 & {$[8.20,9.61]$} & 2.89 \\
\hline & & No & 10.87 & {$[9.78,11.95]$} & 3.80 & 8.59 & {$[8.09,9.10]$} & 2.20 \\
\hline & \multirow{2}{*}{ Long } & Yes & 16.49 & {$[15.40,17.58]$} & 3.58 & 13.49 & {$[12.81,14.17]$} & 3.13 \\
\hline & & No & 16.16 & {$[15.21,17.12]$} & 3.63 & 13.27 & {$[12.55,13.99]$} & 3.44 \\
\hline \multirow{6}{*}{ Reading } & \multirow{2}{*}{ Short } & Yes & 4.34 & {$[3.98,4.70]$} & 1.23 & 3.19 & {$[2.87,3.51]$} & 0.90 \\
\hline & & No & 3.88 & {$[3.50,4.26]$} & 0.68 & 3.09 & {$[2.75,3.43]$} & 0.75 \\
\hline & \multirow{2}{*}{ Med } & Yes & 7.01 & {$[6.59,7.43]$} & 1.78 & 5.09 & {$[4.77,5.41]$} & 1.66 \\
\hline & & No & 6.84 & {$[6.41,7.27]$} & 1.83 & 5.30 & {$[4.93,5.67]$} & 1.54 \\
\hline & \multirow{2}{*}{ Long } & Yes & 10.22 & {$[9.49,10.96]$} & 3.20 & 7.88 & {$[7.42,8.35]$} & 2.23 \\
\hline & & No & 10.67 & {$[9.46,11.88]$} & 3.67 & 7.60 & {$[7.14,8.06]$} & 2.71 \\
\hline
\end{tabular}

Note: The $95 \%$ CI of mean is calculated based on intra-subject standard error.

Figure 3 shows means and inter-subject standard deviations of TSOT with a dashed line at the TSOT grand mean $(7.5 \mathrm{sec})$ as a reference to the average task performance. The letter ' $\mathrm{M}$ ' for male and ' $F$ ' for female are used to label each participant's performance (averaged over the three replications of each task level). Interestingly, the trends in TSOT are largely consistent across the 
different age groups, although the variation between individuals is more pronounced within the 25-39 and 40-54 age groups, especially for text entry tasks.

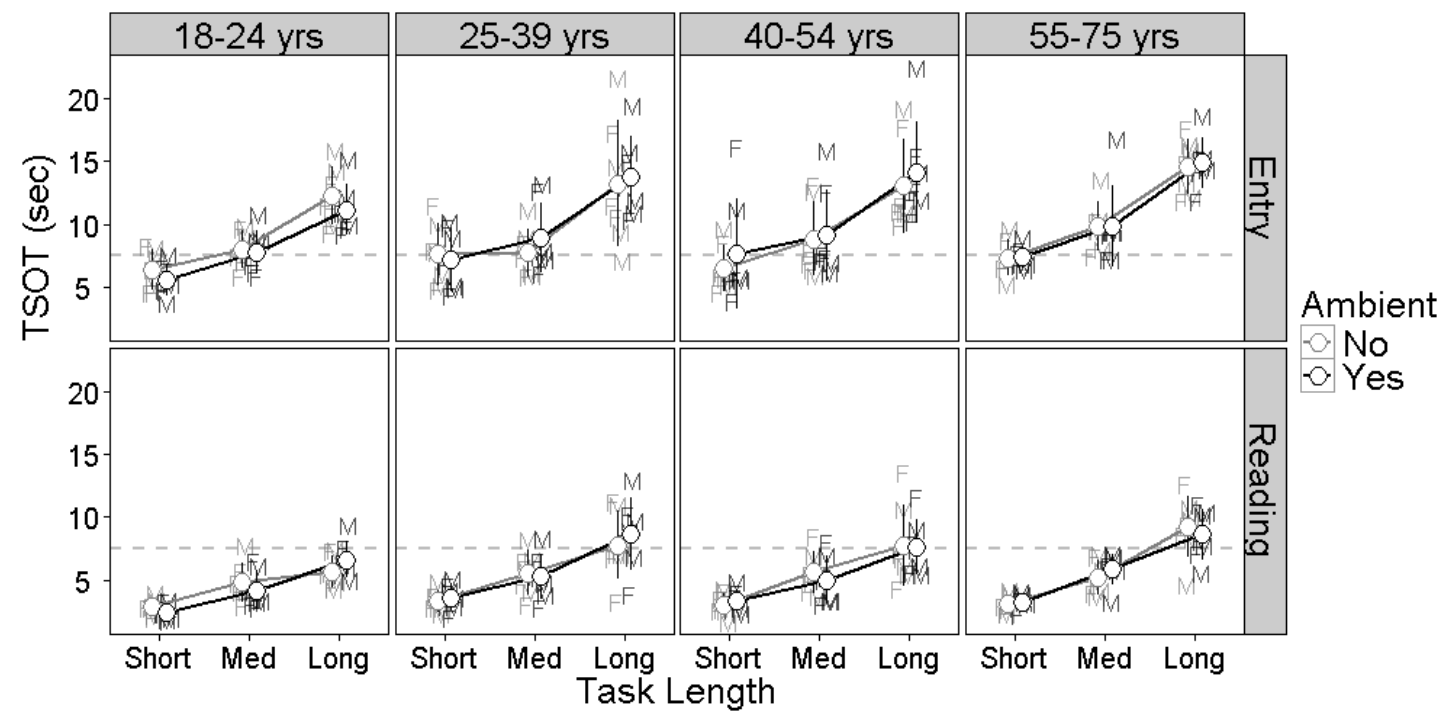

Figure 3. TSOT for occlusion trials by age group for each test condition

\section{Statistical Model of TSOT}

Task type $(F(1,962)=722.91, p<.0001)$ and task length $(F(2,962)=817.04, p<.0001)$ were significant. Specifically, TSOT was larger for text entry compared to text reading and increased when going from short to medium, and from medium to long lengths within each of the task types. The two-way interaction of task type and length was also significant $(F(2,962)=23.66, p$ $<0.0001)$. The increase in TSOT when moving from medium to long entry tasks was larger compared to when moving from medium to long reading tasks (Figure 4, left side).
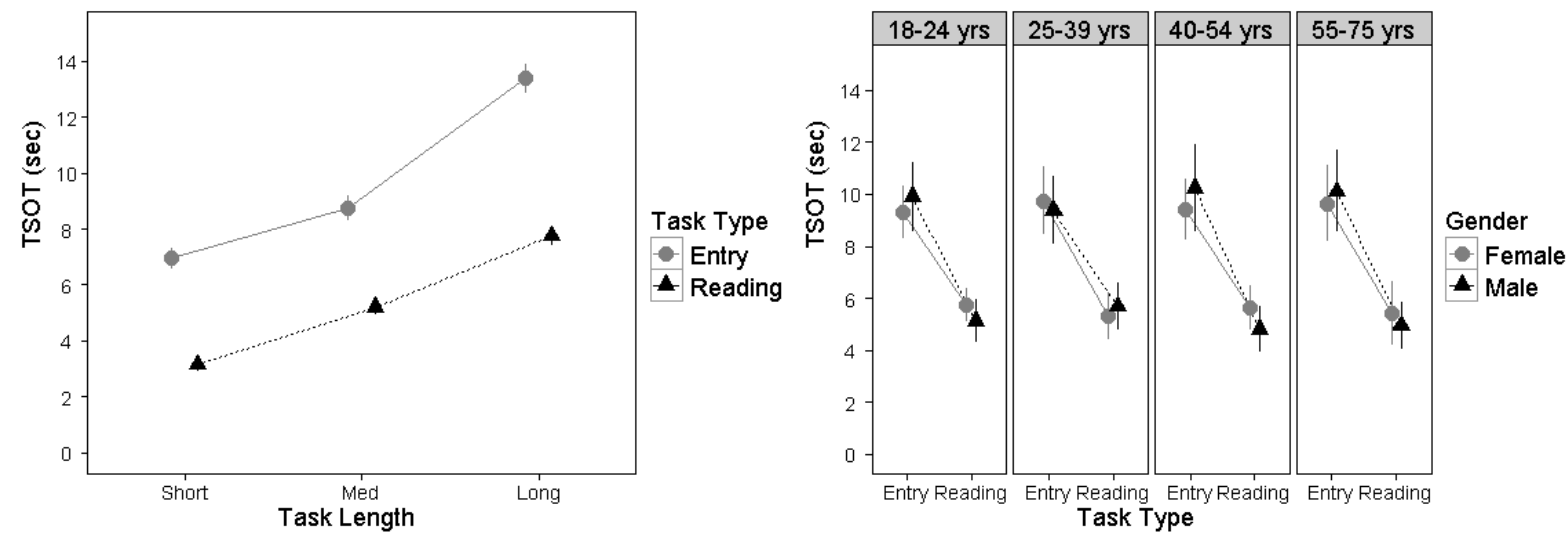

Note: The error bars show the $95 \% \mathrm{CI}$ for mean based on intra-subject standard error.

Figure 4. Interaction plots: task length by task time (left), task type by gender by age (right)

The main effects of ambient text, gender and age were not significant. However, the three-way interaction between task type, age, and gender was significant $(F(3,962)=3.47, p=.02)$. The 25-39-year old age group shows a somewhat different pattern of TSOT for male and female participants (Figure 4, right), i.e., in this group men have a shorter TSOT in text entry and longer 
TSOT in text reading tasks compared to women - the opposite is observed for all other age groups. However, given the small sample size, the effects of gender and age should be interpreted with caution.

\section{DISCUSSION AND CONCLUSION}

The goal of this study was to assess the effect of text reading and text entry could have on driving performance. This study used Total Shutter Open Time (TSOT) as the surrogate measure for the time drivers' eyes would be off the road in completing a task. The results indicated longer TSOTs for entry compared to reading, and an increase in TSOT with longer character lengths in both text reading and entry conditions. For each task level, the total time spent on the task with vision unoccluded in the occlusion trials was generally shorter than the task time in the static condition. This effect was not constant across text reading and entry, demonstrating the need for a surrogate driving task, as a static condition is not sufficient. Contrary to expectations, ambient text had no effect on TSOT. One explanation is that occlusion technique did not require head and eye movements, on and off the screen, to maintain focus on the text box. This is unlike realworld driving and further simulator or on-road studies are needed to validate the outcomes.

Different sources provide conflicting guidelines regarding acceptable TSOT levels; the Japanese Automobile Manufacturer Association (JAMA) sets the limit at $7.5 \mathrm{sec}$ (JAMA, 2004), NHTSA suggests $9.0 \mathrm{sec}$ (NHTSA, 2012), and the Alliance of Automotive Manufacturers suggests 15.0 sec (The Alliance, 2006). These differences substantially impact interpretation of TSOTs. Depending on the limit considered, some conditions may be deemed unsafe for some if not all drivers. It is also important to recognize that differences exist in the way drivers handle text reading and entry tasks of varying levels of difficulty. Accounting for the complexity of the lexicon as well as the continuous structure of text can provide greater insights in this direction. And although the shutter open time interval (vision) used in this study of $1.5 \mathrm{sec}$ is generally agreed upon (Foley, 2008), JAMA and Alliance guidelines suggest a different shutter close time of $1.0 \mathrm{sec}$. This vision-occlusion cycle can influence TSOT. The more more closely it mimics the way drivers distribute their visual attention between the secondary task and the road, the more likely the occlusion technique will produce results that generalize to actual driving.

The occlusion technique is easy to implement and analyze, making it an attractive alternative compared to on-road and simulator studies that require eye tracking and manual coding of video recordings. However, one concern with the occlusion technique is its ability to produce results that can translate to on-road driving. One aspect of such translation relates to a reliable conversion formula between TSOT and total eyes-off-road time (TEORT) - the time on task metric used in simulator and on-road studies (Ranney, Baldwin, Smith, Martin, \& Mazzae, 2012). The results of this study combined with a parallel driving simulator study can provide additional evidence in this direction (Peng, Boyle, Ghazizadeh, \& Lee, 2013).

The occlusion technique has a number of limitations. One critical limitation is that unlike realworld driving, the occlusion technique specifies how often and for how long the vision is occluded, thereby enforcing the pace of glances and preventing long glances. Further, drivers can retain information while vision is not occluded and continue processing while vision is occluded, because there are no other tasks during the occlusion periods. This is not the case with the actual 
timesharing between driving and a secondary task. These limitations suggest that the occlusion technique may need to be modified to better reflect the demands of timesharing between the road and an in-vehicle information display.

\section{ACKNOWLEDGMENTS}

The authors would like to thank Madeleine Gibson and Miralis Torres for their help with experimental material preparation and data collection phases of this project, and the staff at the NADS for development of the in-vehicle task. This material is based upon work supported by the U.S. Dept. of Transportation - National Highway Traffic Safety Administration under Contract No. DTNH22-11-D-00237 (Contracting Officer: Julie Kang). Any opinions, findings, and conclusions or recommendations expressed in this publication are those of the authors and do not necessarily reflect the views of the NHTSA and/or the U.S. DOT.

\section{REFERENCES}

Alliance of Automobile Manufacturers. (2006). Statement of principles, criteria and verification procedures on driver interactions with advanced in-vehicle information and communication systems. Washington, D.C.

Foley, J. P. (2008). Now You See It, Now You Don't: Visual Occlusion as a Surrogate Distraction Measurement Technique. In M. A. Regan, J. D. Lee \& K. L. Young (Eds.), Driver Distraction: Theory, Effects, and Mitigation. Boca Raton, FL: CRC Press.

International Organization for Standardization. (2007). ISO 16673 Road Vehicles-Ergonomic Aspects of Transport Information and Control Systems-Occlusion Method to Assess Visual Demand Due to the Use of In-vehicle Systems. Geneva, Switzerland.

JAMA. (2004). Japan Automobile Manufacturers Association (JAMA) Guideline for In-Vehicle Display Systems, Version 3.0 August.

Lansdown, T. C. (2012). Individual differences and propensity to engage with in-vehicle distractions-A self-report survey. Transportation Research Part F: Traffic Psychology and Behaviour, 15(1), 1-8.

NHTSA. (2012). Visual-Manual Driver Distraction Guidelines Text Reading and Text Input Assessment in Support of the NHTSA Distraction Guidelines. Washington, DC: Department of Transportation.

Peng, Y., Boyle, L. N., Ghazizadeh, M., \& Lee, J. D. (2013). Factors affecting glance behavior when interacting with in-vehicle devices: Implications from a simulator study. Paper presented at the 7th International Driving Symposium on Human Factors in Driver Assessment, Training and Vehicle Design, Bolton Landing, NY.

Ranney, T., Baldwin, S., Smith, L., Martin, J., \& Mazzae, E. (2012). Driver Behavior During Visual-Manual Secondary Task Performance: Occlusion Method Versus Simulated Driving. Washington, D. C.: U.S. DOT: National Highway Traffic Safety Administration.

Senders, J. W., Kristofferson, A., Levison, W., Dietrich, C., \& Ward, J. (1967). The attentional demand of automobile driving. Highway Research Record, 195, 15-33. 